\title{
Covid-19 and Metabolic Syndrome
}

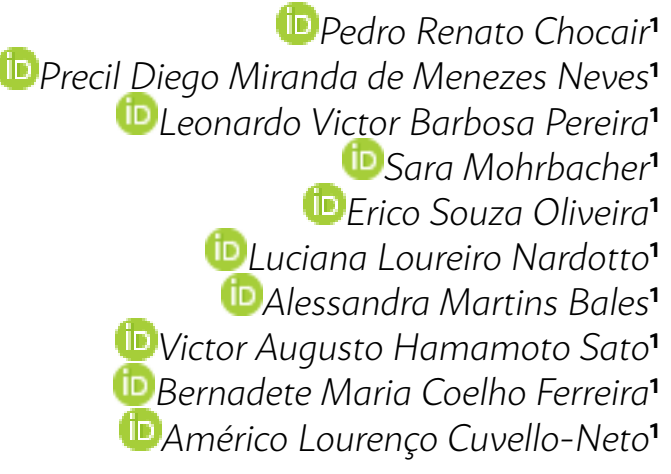

1. Serviço de Clínica Médica. Hospital Alemão Oswaldo Cruz. São Paulo, SP. Brasil.

http://dx.doi.org/10.1590/1806-9282.66.7.871

Dear editors,

In the second half of 2019, a new coronavirus (SarsCov-2) was identified in a city of China, which soon spread to the whole world, becoming a great tragedy and reaching all continents, except for Antarctica. The numbers, widely disclosed, prove the severity of its resulting disease (Covid-19), since over 2 million cases have already been registered throughout the world, with 134,508 deaths by $16 / 04 / 2020$.

After a careful analysis of 43 studies involving 3,600 patients, Fu et al. ${ }^{1}$ pointed out the main signs and symptoms of the disease are: fever (83.3\%), cough (60.3\%), and fatigue (38\%). On the other hand, the most common laboratory alterations were: increased C-reactive protein - PCR (68.6\%), lymphopenia (57.4\%), and increased lactic dehydrogenase - LDH (51.6\%). Groundglass opacities and bilateral pneumonia were the main alterations found in chest CT scans, with frequencies of $80 \%$ and $73 \%$, respectively. They also found that $25.6 \%$ of patients presented severe evolution, reaching lethality in $3.6 \%$ of the cases. The authors concluded that most Covid-19 cases were symptomatic, with a moderate rate of fatality. The residents of Wuhan and those with comorbidities had more severe presentations, with higher mortality.

The severity and, consequently, the lethality and mortality present differences between regions, which is attributed to several factors, such as age and comorbidities. In Italy, $12 \%$ of the cases detected and $16 \%$ of hospitalized patients were admitted to the Intensive Care Unit, with $7.2 \%$ of lethality, according to an analysis carried out in mid-March 2020. In contrast, lethality in South Korea, during the same period, was $0.9 \%^{2-4}$. However less frequent, a severe evolution can also occur in young individuals without apparent comorbidities, but possibly with a subclinical systemic inflammatory state.

The conditions associated with greater severity and, consequently, lethality and mortality are homogeneously recognized in the literature as: obesity, chronic kidney disease, cancer, diabetes mellitus, 
hypertension, cardiovascular disease, and old age $\mathrm{e}^{5-8}$. In other words, greater severity occurs precisely in individuals with metabolic syndrome. Based on this premise, we can better understand the reason for such varying severity in different countries and even in a single country, since the prevalence of the metabolic syndrome is known to be variable. Focusing on the Brazilian scenario, in a sample of the adult population from a city in the south of the Paraná state, a diagnosis of metabolic syndrome was found in approximately $50 \%$ of the population ${ }^{9}$. In another interesting study conducted in Niterói-RJ, Saad et al. ${ }^{10}$ found a high prevalence of metabolic syndrome in the elderly, affecting $64 \%$ of them when analyzed according to the criteria defined by the IDF (International Federation of Diabetes), and 69\% based on the JIS (Joint Interim Statement). That is, very likely, a possible explanation to the higher gravity also among the elderly, considering that the more time of evolution of the metabolic syndrome, the higher the probability of other associated comorbidities.

On the other hand, in an analysis we carried out on 5,000 check-up consultations of an adult population from São Paulo, with a mean age of 42 years, and with a predominantly male population (70\%), we found that $25 \%$ of the individuals met the diagnostic criteria for metabolic syndrome (unpublished data), precisely half of the figure found in Paraná or Niterói ${ }^{910}$.

All these data clearly show the possible relationship between the presence of metabolic syndrome and the severity of Covid-19. Such evidence will certainly be more abundant if we understand that the diagnosis of the metabolic syndrome often precedes the diagnosis of comorbidities. Evidently, those who already have metabolic syndrome with complications will be more susceptible to a worse evolution of Sars-Cov-2.

The variable prevalence of metabolic syndrome should contribute to the different evolution outcomes of the same disease.

To diagnose metabolic syndrome, three or more of the five criteria established by international experts and the World Health Organization need to be met. They are:

- Central obesity: waist circumference equal to or greater than $90 \mathrm{~cm}$ in men and $80 \mathrm{~cm}$ in women, although these numbers vary based on ethnicity. When the BMI is greater than or equal to 30 $\mathrm{kg} / \mathrm{m}^{2}$ there is no need to consider the abdominal circumference.

- Triglycerides: above $150 \mathrm{mg} / \mathrm{dL}$.
- HDL cholesterol: less than $40 \mathrm{mg} / \mathrm{dL}$ in men and $50 \mathrm{mg} / \mathrm{dL}$ in women.

- Fasting glucose: over $100 \mathrm{mg} / \mathrm{dL}$ or diabetes or increased insulin resistance.

- Blood pressure: greater than or equal to 130/85 mmHg or with antihypertensive medication.

(P.S.: increased serum albumin in urine is, for some, a diagnostic criterion and, for others, a sign of kidney involvement in metabolic disease).

The main complications of metabolic syndrome are:

- Atherosclerosis - coronary, cerebral, and others.

- Diabetes type 2 or secondary to increased insulin resistance.

- Arterial hypertension.

- Chronic kidney disease.

- Several types of cancer.

- Other: polycystic ovary syndrome, urinary calculosis, acanthosis nigricans, osteoarthrosis, and hyperuricemia with or without gout, among others.

What is the link between metabolic syndrome and Covid-19? What these diseases have in common? Why is the inflammatory process of coronavirus patients accentuated in those with metabolic syndrome and the elderly?

Adipose cells, as well as the macrophages, endothelial cells, and fibroblasts, are responsible for the production of a series of substances that cause, silently, irreparable damage to our body. Among them, there are adiponectin, interleukin-6, tumor necrosis factor-alpha (TNF-alpha), resistin, leptin, angiotensinogen, and plasminogen activator inhibitor-1 (PAI-1). Adiponectin is reduced in obese patients, and its levels are inversely correlated with insulin resistance and the possible presence of antiatherogenic activity ${ }^{11-14}$.

Interleukin- 6 and TNF-alpha are pro-inflammatory cytokines that can also contribute to insulin resistance and, especially, to the subclinical systemic and continuous inflammatory process responsible for the complications found in patients with the metabolic syndrome, such as diabetes, atherosclerosis, hypertension, and chronic kidney disease. This sustained inflammatory state can be evidenced by an increase in the acute-phase proteins (CRP, fibrinogen, and ferritin).

In addition, the secretion of tissue angiotensinogen may contribute to hypertension, commonly observed in these patients, and to the secretion of PA-1 in a pro-thrombotic state, something that should be considered in patients with metabolic syndrome and Covid-19. 
Therefore, in patients with metabolic syndrome, there is a constant and sustained inflammatory state that is probably exacerbated with the presence of Sars$\mathrm{CoV}-2$, worsening the clinical scenario of Covid-19.

How can the inflammatory state of patients with metabolic syndrome be reduced? This is our proposal because we believe that, if this is successful, cases will be less severe and, consequently, the lethality rate will be lower.

There are two basic ways to handle the coronavirus. One is to fight it directly with vaccines and medicines, but these possibilities are still undergoing studies ${ }^{15.16}$. The other, which is the reason for this analysis, although obvious, has not been highlighted in the literature. It seeks to strengthen those who are most vulnerable, i.e., patients with metabolic syndrome, and there are conditions for this. Time will be an ally, since soon we will have a specific vaccine and, possibly, effective drugs.

As we know, greater severity of the infection by the Sars-CoV-2 is due to increased systemic inflammation, particularly pulmonary, which is demonstrated by a significant elevation of cytokines ("cytokine storm"), in addition to the inflammatory markers such as ferritin, D-dimers, and PCR. In addition, it is possible to observe in these patients a prothrombotic state due to various abnormalities in the hemostatic system, such as platelet dysfunction, impairment of fibrinolysis, and endothelial dysfunction, conditions that are secondary to increased levels of plasminogen activator inhibitor-1, fibrinogen, and factor VIII activity ${ }^{13,16-22}$. These data suggest that patients with metabolic syndrome and Covid-19 should be anticoagulated, due to the prothrombotic state present in both conditions, at least during the period of hospitalization, in the absence of clinical contraindications and in association with all other measures.

There are some ways of reducing the inflammatory state of patients with metabolic syndrome, namely:

\section{CLINICAL CONDUCT}

Metabolic syndrome patients need to be aware of the seriousness of their illness. Perhaps this is an opportune time for changing lifestyles and eating habits. Regular physical activity that is moderate and compatible with the reality of each individual, without exaggeration, brings recognized health benefits.

It is important to emphasize that metabolic syndrome is a potentially serious disease due to all the complications that may arise and do arise from it, often, after a variable period of the syndrome. Therefore, the benefits go far beyond strengthening to handle the new coronavirus.

The improvement of the parameters of metabolic syndrome is not easy, but it starts with a change in lifestyle and with a healthy diet, considering that it is triggered by an excess of food that is harmful to health, unquestionably primarily by carbohydrates, particularly industrialized fructose.

The most recommended diets are the Mediterranean and the Dietary Approaches to Stop Hypertension - DASH, which are mainly poor in sodium and rich in fruits, vegetables, whole grains, and less fatty dairy products, which provide the improvement of several inflammatory parameters ${ }^{23-25}$. In other words, the increased consumption of whole grains, seafood, fish, nuts, seeds, fiber, vegetables, vegetable oils (e.g., olive oil), up to two glasses of red wine per day (with the exception of children and other health conditions), polyunsaturated fatty acids (e.g., krill oil), which are the basis of these two diets, fight the inflammation responsible for the harm caused by metabolic syndrome and reduces mortality due to cardiovascular disease.

If, on the one hand, the Mediterranean and Dash diets are important for the prevention and treatment of metabolic syndrome, on the other, the "western" diet, rich in carbohydrates, particularly fructose, contained in soft drinks, fast-food, 100\% pure and sweet fruit juice, is largely responsible for the high prevalence of obesity, heart disease, and hypertension worldwide. It is important to draw attention that industrialized fructose, usually derived from corn starch, should not be mistaken with that from fruit, known to be beneficial to our health.

Considering the above, we conclude that:

The treatment of metabolic syndrome is an additional alternative for facing the novel coronavirus, but we must not forget that it is much more comprehensive, since it prevents complications that kill much more than the current pandemic.

We suggest that the Ministry of Health, especially Anvisa, classify, as is done in Australia, the quality of food to our health base on a star system visibly displayed on labels. In such a system, for example, on a scale from one to five stars, the best food items for our health would receive five stars, and the worst - which cause of obesity, diabetes mellitus, hypertension and vascular diseases, such as soft drinks, fast-food, and sugar - would receive one star. Certainly, this is more 
appropriate than indicating the percentage of components on labels, since these are difficult to interpret.

A change in lifestyle is crucial. The confinement, with uncontrolled diets in homes and no adequate physical activity, will surely bring an increase of obesity with deleterious consequences for the organism.

The Mediterranean or Dash diet, rich in polyphenols, particularly resveratrol, are of unquestionable importance. We do not recommend the use of resveratrol in capsules or sachets due to the lack of relevant information, such as the risk of its use in patients with comorbidities, the still-unknown risk of drug interactions, in addition to a better definition of dose. However, the current evidence indicates that this product is highly promising in reducing inflammatory cytokines ${ }^{24}$ and it has already been used in various studies in humans, but with small samples, usually obese patients, without concomitant use of other drugs, with positive results and good tolerance.

We recommend quarantine for protecting patients with metabolic syndrome, particularly those with four or five diagnostic criteria.

6. We suggest medication containing metformin and pioglitazone, respectively, 1,000 $\mathrm{mg}$ and $30 \mathrm{mg}$ per day, for all of them, in the absence of contraindications, for a period determined by the assistant physician, based on the clinical and laboratory findings. The recommendation for the use of metformin and pioglitazone is due to the fact that they reduce serum levels of interleukins 6 and 8 in patients with a high insulin resistance ${ }^{25}$. These drugs do not have any effect against the virus, but against the existing inflammation in patients with metabolic syndrome. Therefore, its use is indicated for patients with high insulin resistance, especially before being affected by the novel coronavirus, and should not be used in patients with kidney failure.

\section{REFERENCES}

1. Fu L, Wang B, Yuan T, Chen X, Ao Y, Fitzpatrick T, et al. Clinical characteristics of coronavirus disease 2019; (COVID-19) in China: a systematic review and meta-analysis. J Infect. 2020;80(6):656-65.

2. Wu Z, McGoogan IM. Characteristics of and important lessons from the coronavirus disease 2019 (COVID-19) outbreak in China: summary of a report of 72314 cases from the Chinese center for disease control and prevention. JAMA. 2020. doi: 10.1001/jama.2020.2648.

3. Verity R, Okell LC, Dorigatti I, Winskill P, Whittaker C, Imai N, et al. Estimates of the severity of coronavirus disease 2019: a model-based analysis. Lancet Infect Dis. 2020;20(6):669-77.
We suggest that the isolation period should be maintained until the clinical and laboratory parameters are improved.

In addition, we also suggest prophylactic anticoagulation during the hospitalization period, especially in these patients, in view of the increased risk of thrombosis which, theoretically, they present.

We must emphasize that we had, in no way, with this text, the intention to resolve the tragedy caused by Covid-19, since this can only be accomplished through the work of scientists. Our goal, to be clear, was to warn people and the authorities that there is a widespread disease that should be widely fought and that kills more than Covid-19; it is called metabolic syndrome and, if that was not enough, it is the main responsible for the comorbidities that generate the greater vulnerability to the deleterious effects of Sars-CoV-2. Therefore, to reduce the complications that aggravate the evolution of Covid-19, we need to fight the inflammation that is at the center of metabolic syndrome.

\section{Author's Contribution}

PRC: study design, literature review, preparation of the text and revision of the final version; PDMMN: preparation of the text and revision of the final version; LVBP: preparation of the text and revision of the final version; SM: preparation of the text and revision of the final version; ESO: preparation of the text and revision of the final version; LLN: preparation of the text and revision of the final version; AMB: preparation of the text and revision of the final version; VAHS: preparation of the text and revision of the final version; BMCF: preparation of the text and revision of the final version; ALCN: study design, literature review, preparation of the text and revision of the final version.

4. Grasselli G, Pesenti A, Cecconi M. Critical care utilization for the COVID-19 outbreak in Lombardy, Italy: early experience and forecast during an emergency response. JAMA. 2020. doi: 10.1001/jama.2020.4031.

5. Liu Y, Yan LM, Wan L, Xiang TX, Le A, Liu IM, et al. Viral dynamics in mild and severe cases of COVID-19. Lancet Infect Dis. 2020;20(6):656-7.

6. Liu K, Fang YY, Deng Y, Liu W, Wang MF, Ma JP, et al. Clinical characteristics of novel coronavirus cases in tertiary hospitals in Hubei Province. Chin Med J (Engl). 2020;133(9):1025-31.

7. Onder G, Rezza G, Brusaferro S. Case-fatality rate and characteristics of patients dying in relation to COVID-19 in Italy. JAMA. 2020. doi: 10.1001/ jama.2020.4683. 
8. Liang W, Guan W, Chen R, Wang W, Li J, Xu K, et al. Cancer patients in SARS-CoV-2 infection: a nationwide analysis in China. Lancet Oncol. 2010;21(3):335-7.

9. Bortoletto MSS, Souza RKT, Cabrera MAS, González AD. Síndrome metabólica, componentes e fatores associados em adultos de 40 anos ou mais de um município da Região Sul do Brasil. Cad Saúde Colet. 2016;24(1):32-40.

10. Saad MAN, Cardoso GP, Martins WA, Velarde LGC, Cruz Filho RA. Prevalência de síndrome metabólica em idosos e concordância entre quatro critérios diagnósticos. Arq Bras Cardiol. 2014;102(3):263-9.

11. Grundy SM. Metabolic syndrome update. Trends Cardiovasc Med. 2016;26(4):364-73.

12. Zhang W, Zhao Y, Zhang F, Wang Q, Li T, Liu Z, et al. The use of anti-inflammatory drugs in the treatment of people with severe coronavirus disease 2019 (COVID-19): the perspectives of clinical immunologists from china. Clin Immunol. 2020;214:108393. doi: 10.1016/j.clim.2020.108393.

13. Liu B, Li M, Zhou Z, Guan X, Xiang Y. Can we use interleukin-6 (IL-6) blockade for coronavirus disease 2019 (COVID-19)-induced cytokine release syndrome (CRS)? J Autoimmun. 2020;102452. doi: 10.1016/j.jaut.2020.102452.

14. Leisegang $K$, Henkel $R$, Agarwal A. Obesity and metabolic syndrome associated with systemic inflammation and the impact on the male reproductive system. Am J Reprod Immunol. 2019;82(5):e13178.

15. Sanders JM, Monogue ML, Jodlowski TZ, Cutrell JB. Pharmacology treatments for coronavirus disease 2019 (COVID-19): a review. JAMA. 2020 doi: 10.1001/jama.2020.6019.

16. Kostapanos MS, Florentin M, Elisaf MS, Mikhailidis DP. Hemostatic factors and the metabolic syndrome. Curr Vasc Pharmacol. 2013;11(6):880-905.

17. Alessi MC, Juhan-Vague I. Metabolic syndrome, haemostasis and thrombosis. Thromb Haemost. 2008;99(6):995-1000.

18. Kraja AT, Province MA, Arnett D, Wagenknecht L, Tang W, Hopkins PN, et al. Do inflammation and procoagulation biomarkers contribute to the metabolic syndrome cluster? Nutr Metab (Lond). 2007;4:28.

19. Samad F, Ruf W. Inflammation, obesity, and thrombosis. Blood. 2013;122(20):3415-22.

20. Rao AK, Freishtat RI, Jalagadugula G, Singh A, Mao G, Wiles A, et al. Alterations in insulin-signaling and coagulation pathways in platelets during hyperglycemia-hyperinsulinemia in healthy non-diabetic subject. Thromb Res. 2014;134(3):704-10.

21. Ay C, Tengler T, Vormittag R, Simanek R, Dorda W, Vukovich T, et al. Venous thromboembolism: a manifestation of the metabolic syndrome. Haematologica. 2007;92(3):374-80.

22. Vaduganathan M, Alviar CL, Arikan ME, Tellez A, Guthikonda S, DeLao T, et al. Platelet reactivity and response to aspirin in subjects with the metabollic syndrome. Am Heart J. 2008;156(5):1002.e1-1002.e7.

23. Welty FK, Alfaddagh A, Elajami TK. Targeting inflammation in metabolic syndrome. Transl Res. 2016;167(1):257-80.

24. Coutinho DS, Pacheco MT, Frozza RL, Bernardi A. Anti-inflammatory effects of resveratrol: mechanistic insights. Int J Mol Sci. 2018;19(6):1812.

25. Ali DS, Shah M, Ali A, Malik MO, Rehman F, Badshah H, et al. Treatment with metformin and combination of metformin plus pioglitazone on serum levels of IL-6 and IL-8 in polycystic ovary syndrome: a randomized clinical trial. Horm Metab Res. 2019;51(11):714-22. 